Check for updates

Cite this: RSC Adv., 2018, 8, 21363

Received 26th March 2018

Accepted 28th May 2018

DOI: $10.1039 / c 8 r a 02621 a$

rsc.li/rsc-advances

\section{Anionic polyelectrolytes in titanosilicate molecular sieve synthesis towards simultaneously accomplishing low production cost and high catalytic activity $\uparrow$}

\begin{abstract}
Kairui Fu, Jingui Wang, (D) Yichen Wang, Yuanchao Shao, Jiaqi Zhu and Tianduo Li*
Assisted by an anionic polyelectrolyte of poly(acrylic acid) (PAA), high-performance titanium silicalite-1 (TS1) could be facilely synthesized at very low usage of expensive organic templates (tetrapropylammonium hydroxide). The presence of PAA helped to incorporate more active Ti species into the TS-1 framework and change the morphology to a plate-like shape, which was beneficial to molecular diffusion among its micropores to access the Ti active sites. Therefore, TS-1 synthesized with PAA showed much higher catalytic activity than that synthesized using the traditional synthesis without polyelectrolytes, and only a $30 \%$ usage amount of organic template was used. Moreover, this ultra-cheap catalyst also displayed a better catalytic activity than commercial TS-1 synthesized via a series of complicated preparation processes including alkene epoxidation with hydrogen peroxide as a green oxidant.
\end{abstract}

\section{Introduction}

As a successful typical material used in the eco-chemical industry, titanium silicate-1 (TS-1) receives much attention and is very valuable because of its unique catalytic performance combined with $\mathrm{H}_{2} \mathrm{O}_{2}$ as an oxidant. TS-1 was proven to simultaneously realize environmental and economic profit in alkene epoxidation, ${ }^{1-8}$ ketone ammoximation ${ }^{9,10}$ and aromatics hydroxylation. ${ }^{11}$ Furthermore, TS-1 has also shown high catalytic performance in alkane oxidation, ${ }^{12}$ oxidation desulfurization $^{13,14}$ and so on. ${ }^{15-18}$ However, due to the very harsh preparation process and extremely expensive raw materials, the production cost of TS-1 is very high, which has hindered its widespread application. So, reducing the production cost has been a hot research area since the first discovery of this milestone catalyst.

TS-1 was first synthesized in 1983 using tetrapropylammonium hydroxide (TPAOH) as an organic template, tetraethyl orthosilicate (TEOS) as a silicon source and tetraethyl orthotitanate (TEOT) as a titanium source. ${ }^{19}$ This method is called the traditional synthetic method. However, this method requires a large amount of highly pure TPAOH that is strictly free of alkali metal ions, which is the main reason for the high cost of TS-1. Actually, in the traditional synthesis, the contribution of

Shandong Provincial Key Laboratory of Fine Chemicals, School of Chemistry and Pharmaceutical Engineering, Qilu University of Technology (Shandong Academy of Sciences), Jinan 250353, P. R. China. E-mail: JGWang@qlu.edu.cn; ylpt6296@vip. $163 . \mathrm{com}$

$\dagger$ Electronic supplementary information (ESI) available: SEM images. See DOI: $10.1039 / \mathrm{c} 8 \mathrm{ra0} 2621 \mathrm{a}$ the TPAOH template was more than $96 \%$ of the total cost of the raw materials. Although many researchers chose other cheap templates to reduce the production cost, including tetrapropylammonium bromide (TPABr) $)^{7,20-26}$ with the same cation $\mathrm{TPA}^{+}$ as TPAOH or a mixture of tetraethylammonium chloride (TEACl) and tetrabutylammonium chloride (TBACl) ${ }^{27}$ the catalytic performance was not satisfactory due to the low number of active Ti centers and the large TS- 1 crystal size. Therefore, many efforts have focused on developing novel strategies to reduce the production cost by decreasing the usage amount of TPAOH to as low as possible, such as temperature-programmed hydrothermal crystallization, controlled multi-hydrolysis processes and solid-phase crystallization. ${ }^{28-31}$ These strategies were proven to significantly reduce the usage of ТРAOH. However, they all required multiple-step synthetic processes, which would significantly increase the production cost including extra equipment and energy. Besides, these methods were complicated and required the cautious operation of matching the hydrolysis and crystallization rates of the Si and Ti sources, which caused difficulty in practical production. This greatly decreased the production reproducibility, leading to a further increase in the production cost. Therefore, a facile and direct route aimed at high-performance and low-cost TS- 1 using a small amount of expensive TPAOH template is greatly desired.

In our previous study ${ }^{32}$ we discovered that poly(acrylic acid) could adjust the crystallization kinetics and modify the crystal size of the titanosilicate molecular sieves to achieve a highactivity titanosilicate catalyst. Here, we employed this unique anionic polyelectrolyte in the low-cost synthesis of TS-1 titanosilicate at a low usage of TPAOH by a one-pot synthesis and 
compared the catalytic performance with TS- 1 synthesized using the traditional synthesis method and current commercial TS-1 catalysts synthesized via a complicated multiple-step synthesis and post-treatments.

\section{Experimental}

\section{TS-1 synthesis}

Firstly, titanium tetra- $n$-butoxide(TBOT) was added to hydrogen peroxide aqueous solution, a clear yellow solution was obtained after stirring for 30 minutes and then the tetrapropylammonium hydroxide(TPAOH) was added under stirring. Thereafter, tetraethyl orthosilicate (TEOS) as the silicon source was added to the solution and the solution was stirred overnight to fully hydrolyze. Then, the solution was heated to $353 \mathrm{~K}$ to remove alcohol produced by hydrolysis. After cooling down, poly(acrylic acid) (PAA) was added under stirring. The molar composition of the mixture was: $\mathrm{SiO}_{2} /$ $\mathrm{TiO}_{2} / \mathrm{TPAOH} / \mathrm{H}_{2} \mathrm{O} / \mathrm{PAA}$ (unit) $=1: 0.025: x: 30: y$. The mixture was transferred into an autoclave and treated at $443 \mathrm{~K}$ for 2 days under static conditions. After treatment, the solid was obtained by filtration or centrifugation, dried at $353 \mathrm{~K}$ overnight, and calcined at $823 \mathrm{~K}$ for $6 \mathrm{~h}$ to remove the organic templates. The samples were named TS-1- $x-y$, where $x$ and $y$ are the amount of TPAOH and PAA added. For example, TS-1-0.15-0.05 means the molar ratio of $\mathrm{SiO}_{2} /$ TPAOH was $1: 0.15$ and $\mathrm{SiO}_{2} / \mathrm{PAA}($ unit) was all $1: 0.05$.

\section{Characterization}

Powder X-ray diffraction (PXRD) was carried out using a Bruker Powder D8 Advance diffractometer operated at $40 \mathrm{kV}$ and $40 \mathrm{~mA}$ with $\mathrm{CuK} \alpha$ radiation $(\lambda=1.5418$ Angstrom). Diffuse reflectance ultraviolet-visible (DRUV/vis) spectra were measured on a Shimadzu UV-2450 spectrophotometer at $298 \mathrm{~K}$ with $\mathrm{BaSO} 4$ as a reference. IR spectra were measured on a Shimadzu IRPrestige-21 spectrometer as $\mathrm{KBr}$ pellets. Elemental analyses ( $\mathrm{Si}$ and $\mathrm{Ti}$ ) were measured on an inductively coupled plasma optical emission spectrometer (ICP-OES, Perkin Elmer ICP Optima 2000DV). Field-emission scanning electron microscope (SEM) images were recorded on a JEOL JSM-7600F microscope at $5 \mathrm{kV}$. Transmission electron microscopy (TEM) observations were performed using a JEOL JEM-1400 TEM microscope working at $100 \mathrm{kV}$. Particle sizes were measured on Malvern Zetasizer Nano ZS90 analyzer.

\section{Catalytic reactions}

The oxidation reactions were performed with catalyst (25 mg), 1hexene $(5 \mathrm{mmol})$ and $\mathrm{H}_{2} \mathrm{O}_{2}(5 \mathrm{mmol})$ in methanol $(5 \mathrm{ml})$ in a $20 \mathrm{ml}$ glass reactor with a $333 \mathrm{~K}$ oil bath with stirring for $2 \mathrm{~h}$. After the reaction, the mixture was analyzed by gas chromatography. The $\mathrm{H}_{2} \mathrm{O}_{2}$ was determined with a standard $\mathrm{Ce}\left(\mathrm{SO}_{4}\right)_{2}$ solution $(0.1 \mathrm{M})$.

\section{Results and discussion}

The synthetic composition and porosity of the synthesized TS-1 samples are summarized in Table 1 . The starting $\mathrm{Si} / \mathrm{Ti}$ ratio was fixed at 40, which was the theoretical possible maximal $\mathrm{Ti}$ content incorporated into the TS- 1 catalysts. ${ }^{33}$ Considering the usage amount of TPAOH in the traditional synthesis was 0.45 in molar ratio to $\mathrm{SiO}_{2}$, herein, the amount of TPAOH was gradually reduced to 0.15 , which is only about $30 \%$ of the usage amount in the traditional synthesis. Meanwhile, PAA was added to adjust the crystallization kinetics to achieve high catalytic activity. ${ }^{32}$ Moreover, assisted by PAA, the yield of TS-1 was almost $100 \%$. The total titanium source in the synthetic gel was presented in the final $\mathrm{TS}-1$, leading to a $\mathrm{Si} / \mathrm{Ti}$ ratio of approximately 40 . The yield of sample TS-1-0.15-0 was only $82 \%$ and the Ti content in TS-1-0.15-0 was slightly less than that of the samples synthesized with PAA. Compared to the traditional synthetic method where $\mathrm{TPAOH} / \mathrm{SiO}_{2}=0.45$, a yield of about $70 \%$ and an $\mathrm{Si} / \mathrm{Ti}$ ratio of 51 could be achieved (TS-1-0.450 sample).

The nitrogen adsorption characterization (Fig. 1B) showed all the samples had type I adsorption-desorption isotherms, indicating a microporous structure. In addition, more micropores (volume) were present in TS- 1 synthesized with PAA than that from the traditional synthesis (Table 1). The external surface area has a strong relationship with the crystal size. The larger the external surface area is, the smaller the crystal size. Usually, for a microporous catalyst, small-sized crystals possess short microporous channels, which show better catalytic performance due to the easy diffusion of reactants among these short micropores. Comparing TS-1-0.15-0.15, TS-1-0.15-0.10 and TS-1-0.15-0.05, sample TS-1-0.15-0.05 showed the highest external surface area among the samples synthesized with PAA, implying that is has the smallest crystal size. Sample TS-1-0.15-

Table 1 Synthetic composition and porosity of the synthesized TS-1 samples

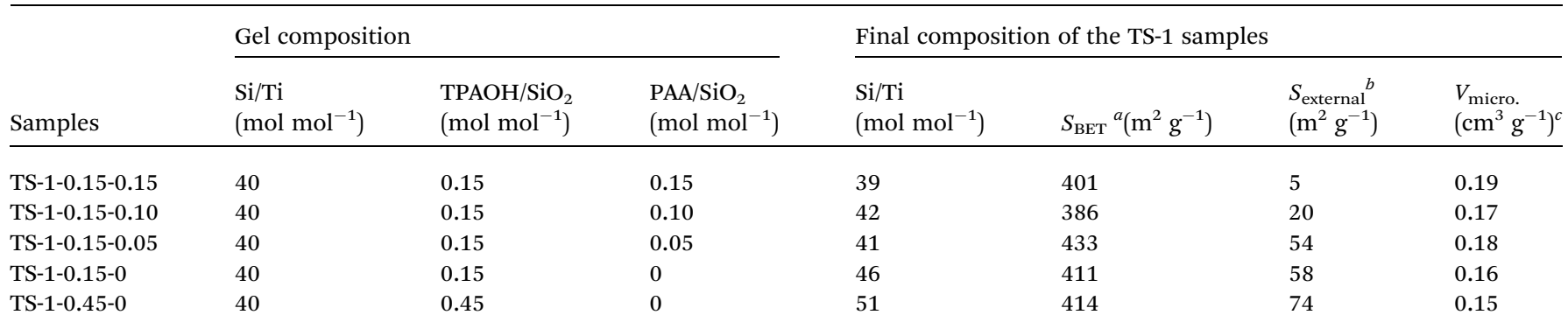

${ }^{a}$ Brunauer-Emmett-Teller (BET) surface area estimated by nitrogen adsorption/desorption measurements. ${ }^{b}$ External surface area calculated by $t$-plot curves. ${ }^{c}$ Micropore volume. 

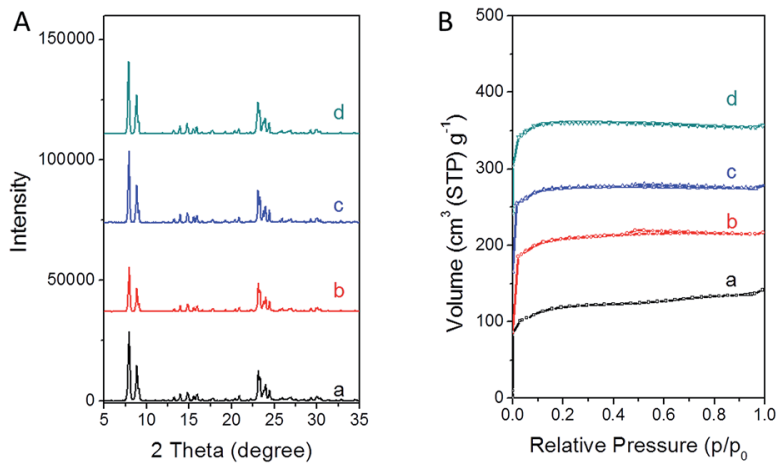

Fig. 1 PXRD patterns and nitrogen adsorption/desorption isotherms of the final TS-1 products (a) TS-1-0.15-0, (b) TS-1-0.15-0.05, (c) TS-1$0.15-0.10$, and (d) TS-1-0.15-0.15.

0 synthesized without PAA showed slightly higher external surface area than that of sample TS-1-0.15-0.5, indicating the smaller size of TS-1-0.15-0. It should be noted that sample TS-10.45-0 from the traditional synthetic method using a large amount of TPAOH had the largest external surface area among all the TS-1 samples here, indicating the smallest crystal size.

Fig. 1A shows the PXRD patterns of TS-1 synthesized with different amounts of the TPAOH template and the PAA polyelectrolyte. All the samples show characteristic peaks of the MFI-type structure. No other excess peaks were observed, indicating the pure crystallization phase. The low diffraction intensity of sample TS-1-0.15-0.05 was probably due to the noncrystalline raw materials and/or the small crystal size.

As shown in Fig. 2 and Fig. 3, the SEM and TEM images show that TS-1-0.15-0 synthesized without PAA has an ellipsoidal shape with a dimeter of about $0.6 \mu \mathrm{m}$. With the increase in the usage amount of PAA, the crystal size of the obtained TS-1 samples increased gradually. Significantly, the morphology changed from spherical to plate-like with uniform thickness. The spherical TS-1-0.15-0.05 sample with a diameter of about 1 micrometer was obtained, which was larger than TS-1-0.15-0.
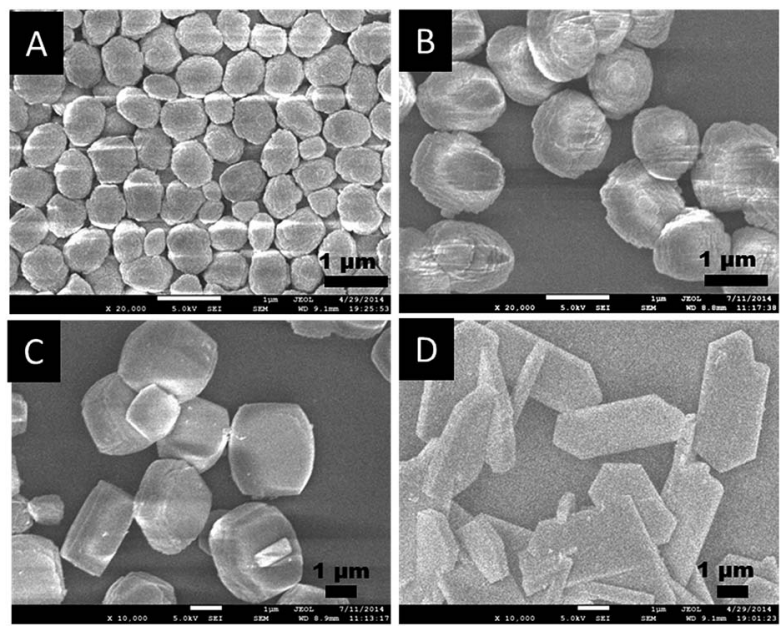

Fig. 2 SEM images of the final TS-1 products (A) TS-1-0.15-0, (B) TS-10.15-0.05, (C) TS-1-0.15-0.10, and (D) TS-1-0.15-0.15.
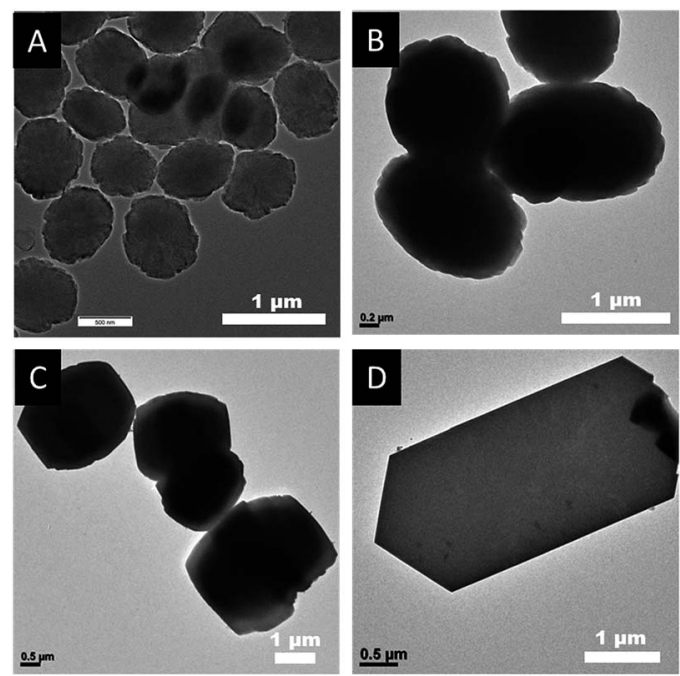

Fig. 3 TEM images of final the TS-1 products (A) TS-1-0.15-0, (B) TS1-0.15-0.05, (C) TS-1-0.15-0.10, and (D) TS-1-0.15-0.15.

The plate-like sample of TS-1-0.15-0.10 $(3.0 \times 2.5 \times 1.0 \mu \mathrm{m})$ had a uniform thickness of about $1 \mu \mathrm{m}$. Further increasing the amount of PAA, the plate-like sample of TS-1-0.15-0.15 (5.0 $\times$ $2.5 \times 0.6 \mu \mathrm{m})$ displayed thinner thickness than that of TS-10.15-0.10. This characteristic implies that these TS- 1 products are still potential high-performance catalysts although they have a large size in the other two dimensions. ${ }^{34}$ It should be noted that TS-1-0.45-0 synthesized by traditional methods had the smallest particle size (100-200 nm) among these TS-1 samples, indicating the traditional method is good at producing small sized TS-1 catalysts.

Interestingly, as shown in the enlarged SEM and TEM images (Fig. 4), the TS-1-0.15-0.05 sample had a rough surface morphology comprised of merged nano-crystals. This may
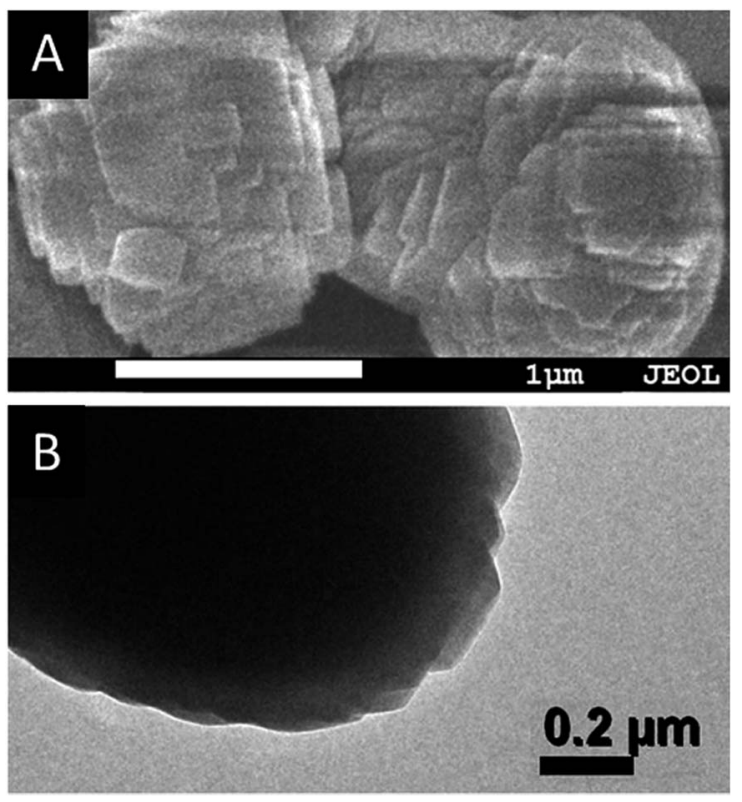

Fig. 4 The enlarged (A) SEM and (B) TEM images of TS-1-0.15-0.05. 
further contribute to its external surface area and further decrease the mass transportation restriction. In addition, the SEM image in Fig. 2B and the TEM image in Fig. 3B revealed that TS-1-0.15-0.05 was completely crystallized without any amorphous raw materials, which suggests that the low intensity in the above PXRD pattern was probably due to these nanocrystals.

For microporous titanosilicates, besides the molecular diffusion restriction in the micropores, the Ti content and state are also important factors for the catalytic reaction. It has been found that there are several different states of $\mathrm{Ti}$ species in titanosilicate, including tetracoordinated, hexacoordinated, oligomeric Ti species and small-sized $\mathrm{TiO}_{2}$ particles. However, only isolated tetra-coordinated Ti species, namely framework Ti species, have been proven to provide the catalytic activity. ${ }^{19,35-38}$ The coordination state of the Ti species in TS- 1 can be detected by DRUV/Vis and FTIR spectroscopy.

As shown in Fig. 5A, all the samples show a main peak at $210 \mathrm{~nm}$, indicating that Ti species are present in the framework. There is a shoulder peak at $270 \mathrm{~nm}$ and a small peak at around $330 \mathrm{~nm}$ for the sample of TS-1-0.15-0 synthesized without PAA, suggesting that there are significant extra-framework titanium species in this sample. For the samples synthesized with PAA, TS-1-0.15-0.10 shows a shoulder peak at around $270 \mathrm{~nm}$ related to the hexacoordinated Ti species. TS-1-0.15-0.15 shows another band at about $330 \mathrm{~nm}$ besides $270 \mathrm{~nm}$, indicating the formation of hexacoordinated $\mathrm{Ti}$ species and anatase-like $\mathrm{TiO}_{2}$ tiny particles. Importantly, TS-1-0.15-0.05 almost shows a peak at $210 \mathrm{~nm}$ related to the framework Ti species. Considering a near 100\% yield was achieved assisted by PAA and a maximal possible titanium content in the starting gel ( $\mathrm{Si} / \mathrm{Ti}$ molar ratio of 40 ), it was exciting to find that the sample of TS-1-0.15-0.05 directly achieved the maximal framework titanium content without requiring an excess amount of titanium starting sources. In addition, TS-1 synthesized by the traditional method displayed a slightly lower intensity band at $210 \mathrm{~nm}$ as compared to TS-1 assisted by PAA, suggesting a low amount framework titanium species.

The framework titanium in TS-1 titanosilicate could also be observed by the FTIR technique. A band at $960 \mathrm{~cm}^{-1}$ in the FTIR spectrum is usually an indication of isomorphous substitution of Ti in the TS-1 lattice. The intensity of this band increases proportionally with an increase in framework Ti content. As shown in Fig. 5B, all the samples show the band at $960 \mathrm{~cm}^{-1}$, giving further evidence of framework $\mathrm{Ti}$ in all the samples. The intensity of this band was similar for all the TS-1 samples synthesized with the assistance of PAA, which is higher than that of TS-1-0.15-0 synthesized using the traditional method (Fig. 5C). This indicates that PAA can help to incorporate more framework Ti species into TS-1 zeolites. However, the effect of PAA did not directly help Ti to insert into the framework. The mechanism was based on the adjustment of the crystallization mechanism as previous reported. ${ }^{32}$ As a gelating agent, PAA could turn the starting solution to a solid gel, which could result in a solid-phase transformation from the liquid-phase mechanism. It was proven that the best conditions to reach the highest Ti content without extra-framework Ti species was the synergistic effect of the solid-phase and the liquid-phase transformation. Therefore, the optimal amount of PAA should form a mixture composed of both liquid-phase and solid-phase precursors. Here, the sample of TS-1-0.15-0.05 achieved the optimal addition amount of PAA. The samples of TS-1-0.15-0.10 and TS-1-0.15-0.15 also had a higher content of framework Ti species than that of sample TS-1-0.15-0, although some extraframework and/or anatase-like Ti species were also present.

In addition, a band at $550 \mathrm{~cm}^{-1}$ in the FTIR spectrum (Fig. 5B) is characteristic of the vibration of the five-membered ring of MFI structure. All the samples show the band at $550 \mathrm{~cm}^{-1}$, suggesting that all the samples had MFI type structures. This result was consistent with the XRD results.

The epoxidation of alkenes is a greatly important industrial process for producing epoxides. Here, the catalytic oxidation of
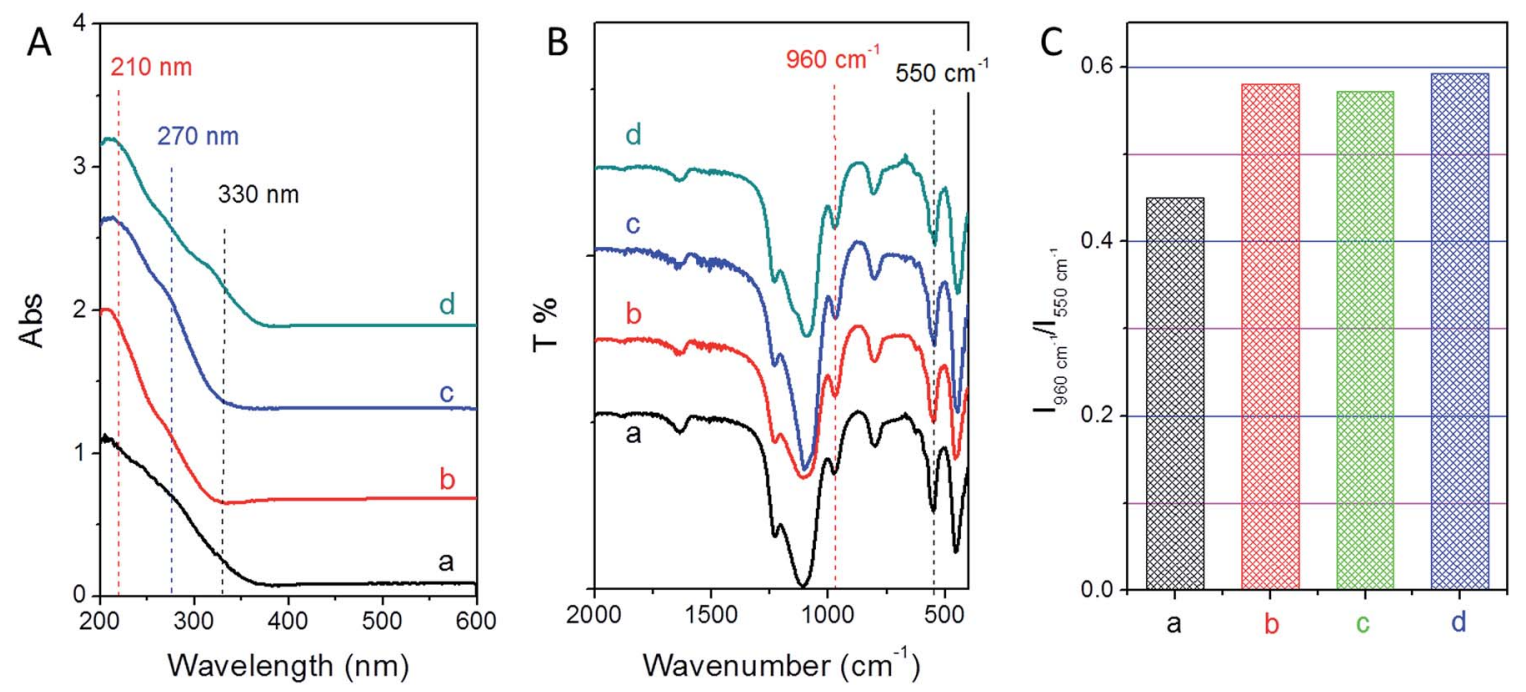

Fig. 5 (A) DRUV-vis spectra, (B) FTIR spectra and (C) intensity ratio of $960 \mathrm{~cm}-1$ to $550 \mathrm{~cm}-1$ of the final TS-1 products (a) TS-1-0.15-0, (b) TS-10.15-0.05, (c) TS-1-0.15-0.10, and (d) TS-1-0.15-0.15. 
Table 2 Catalytic oxidation of 1-hexene using various TS-1 catalysts ${ }^{a}$

\begin{tabular}{|c|c|c|c|c|c|c|}
\hline Samples & $\mathrm{Si} / \mathrm{Ti}\left(\mathrm{mol} \mathrm{mol}{ }^{-1}\right)$ & Crystal size $(\mu \mathrm{m})$ & Conv. (\%) & $S_{\text {epoxide }}^{b}(\%)$ & $E_{\mathrm{H}_{2} \mathrm{O}_{2}}(\%)^{c}$ & $\mathrm{TON}^{d}\left(\mathrm{~mol} \mathrm{~mol}^{-1}-\mathrm{Ti}\right)$ \\
\hline TS-1-0.15-0 & 46 & $\sim 0.6$ spherical & 9.1 & 93.1 & 71 & 52 \\
\hline TS-1-0.15-0.05 & 41 & $\sim 1.0$ spherical & 26.5 & 93.4 & 84 & 135 \\
\hline TS-1-0.15-0.15 & 39 & $5.0 \times 2.5 \times 0.6$ & 24.3 & 94.6 & 94 & 118 \\
\hline TS-1-0.45-0 & 51 & $0.1 \times 0.1 \times 0.1$ & 12.7 & 97.2 & 69 & 80 \\
\hline TS- $1^{e}$ & 45 & $\sim 0.3$ spherical & 24.4 & 90.8 & 80.2 & 136 \\
\hline
\end{tabular}

${ }^{a}$ Reaction conditions: catalyst $(25 \mathrm{mg})$, methanol $(5 \mathrm{ml})$, 1-hexene $(5 \mathrm{mmol})$, and $\mathrm{H}_{2} \mathrm{O}_{2}(5 \mathrm{mmol}), 60{ }^{\circ} \mathrm{C}, 2$ h. ${ }^{b}$ Selectivity of the epoxide. ${ }^{c}$ Utilization efficiency of $\mathrm{H}_{2} \mathrm{O}_{2}$ toward the oxidation of 1-hexene. ${ }^{d}$ Turnover number per Ti site. ${ }^{e}$ Commercial TS-1 from the Catalysis Society of Japan.

1-hexene in the presence of $\mathrm{H}_{2} \mathrm{O}_{2}$ was chosen to evaluate the catalytic performance of the synthesized TS- 1 catalysts and their catalytic performance was compared with TS-1 from the traditional synthesis method and current commercial TS-1 catalysts.

The catalytic results are summarized in Table 2. All the TS-1 samples assisted by PAA showed better catalytic performance than the TS-1-0.15-0 sample synthesized by the traditional synthesis. Although the amount of ТРAOH to silica was increased to 0.45 , the TS-1-0.45-0 sample still showed lower catalytic activity than that of TS- 1 synthesized with PAA. Here, the crystal size of the TS- 1 samples from the traditional synthesis was smaller than that of those synthesized with PAA. The lower catalytic performance of TS- 1 from the traditional synthesis may be attributed to the lower framework Ti content as discussed above. The lower turnover numbers (TON) of TS-10.15-0 and TS-1-0.45-0 also implied some of the Ti species in the samples were inert for catalytic performance.

It has been reported that there will be no obvious diffusion restriction when a crystal size less than $1 \mu \mathrm{m}$ is used for 1hexene oxidation. ${ }^{39}$ Here, comparing plate-like TS-1-0.15-0.15 $(5.0 \times 2.5 \times 0.6 \mu \mathrm{m})$ and plate-like TS-1-0.15-0.10 $(3.0 \times 2.5 \times$ $1.0 \mu \mathrm{m})$, they showed similar 1-hexene conversion and similar turnover numbers (TON) despite different crystal sizes and thicknesses, which supports the above-mentioned conclusion. In addition, assisted by PAA, TS- 1 crystals less than $1 \mu \mathrm{m}$ in length in one dimension (plate-like) or three dimensions (spherical) could be obtained. It was found that 2-dimensional plate-like crystals (TS-1-0.15-0.10 and TS-1-0.15-0.15) showed slightly lower catalytic conversion of 1-hexene and lower TON than spherical crystals with a small size in 3 dimensions (TS-10.15-0.05), indicating lower diffusion restriction of spherical small crystals. Meanwhile, it is implied that a high-activity TS-1 catalyst could be achieved if the diffusion length of the crystal was reduced to less than $1 \mu \mathrm{m}$ in one dimension.

It should be noted that TS-1-0.45-0 from the traditional synthesis showed lower 1-hexene conversion although it had a small size $(0.1 \times 0.1 \times 0.1 \mu \mathrm{m}$ as shown in Fig. S1 $\dagger)$. It would be very easy to transport reactants in such a small crystal. The reason for the low conversion is the low number of active Ti centers as indicated by the low TON, which may be the intrinsic disadvantage of the traditional synthetic system. For commercial TS-1 catalysts with $\sim 300 \mathrm{~nm}$ sized crystals (Fig. S1†), complicated post-treatment processes were still required to improve the catalytic ability of the Ti centers. ${ }^{40-42}$ The TON of commercial TS-
1 could reach 136, which is much higher than the TON (80) of TS1 directly synthesized using the traditional system. However, post-treatment would leach the Ti species from the TS- 1 framework, leading to a lower framework Ti content. Comparing TS-10.15-0.05 and commercial TS-1, both had a similar TON, indicating the similar catalytic activity of each Ti species. However, TS-1-0.15-0.05 showed a slightly higher conversion of 1-hexene attributed to the higher Ti content. In addition, complicated post-treatment would further increase the production cost due to the usage of new agents and excess energy. Therefore, it was really significant to directly achieve an ultra-low-cost TS-1 catalyst with a high Ti content, high Ti activity and a high yield, which showed great commercial potential.

\section{Conclusions}

We have successfully synthesized a series of low-cost TS-1 titanosilicate molecular sieves by cutting the usage of tetrapropylammonium hydroxide (TPAOH) and assisted with polyelectrolyte PAA. Plate-like and spherical crystals were obtained via adjusting the amount of PAA. High-performance TS-1 could still be obtained when the usage amount of TPAOH was reduced by about $70 \%$. PAA played important roles in the synthesis: (1) to change the crystal morphology to achieve less diffusion restriction in one or more dimensions; (2) to directly achieve the maximal framework Ti content (2.0 wt\%); (3) to achieve a near $100 \%$ yield. The obtained TS- 1 catalysts assisted by PAA showed much higher catalytic activity $(\mathrm{TON}=118-135)$ than that from the traditional synthesis $(\mathrm{TON}=80)$. TS- 1 from one-pot synthesis assisted with PAA without any other posttreatment steps still showed slightly higher catalytic activity than commercial TS-1 from a series of complicated preparation processes, suggesting promising commercial potential.

\section{Conflicts of interest}

There are no conflicts to declare.

\section{Acknowledgements}

This work is supported by the National Natural Science Foundation of China (No. 51602164, 21376125), and the Program for Scientific Research Innovation Team in Colleges and Universities of Shandong Province. 


\section{Notes and references}

1 M. G. Clerici, G. Bellussi and U. Romano, J. Catal., 1991, 129, 159-167.

2 S. B. Kumar, S. P. Mirajkar, G. C. G. Pais, P. Kumar and R. Kumar, J. Catal., 1995, 156, 163-166.

3 D. Serrano, R. Sanz, P. Pizarroa and I. Morenoa, Chem. Commun., 2009, 1407-1409.

4 J. Wang, L. Xu, K. Zhang, H. Peng, H. Wu, J.-g. Jiang, Y. Liu and P. Wu, J. Catal., 2012, 288, 16-23.

5 L. Wang, J. Sun, X. Meng, W. Zhang, J. Zhang, S. Pan, Z. Shen and F. S. Xiao, Chem. Commun., 2014, 50, 2012-2014.

6 J. Kim, J. Chunab and R. Ryoo, Chem. Commun., 2015, 51, 13102-13105.

7 Y. Zuo, M. Liu, T. Zhang, C. Meng, X. Guo and C. Song, ChemCatChem, 2015, 7, 2660-2668.

8 M. Lin, C. Xia, B. L. Zhu and X. Shu, Chem. Eng. J., 2016, 295, 370-375.

9 J. Le Bars, J. Dakka and R. A. Sheldon, Appl. Catal., A, 1995, 136, 69-80.

10 T. Zhang, Y. Wang, S. Wang, X. Wu, P. Yao, Y. Lin and J. Xu, React. Kinet., Mech. Catal., 2015, 114, 735-752.

11 M. Lin, X. T. Shu, X. Q. Wang and B. Zhu, China patent, 99126289.1, 1999.

12 D. R. C. Huybrechts, L. DeBruycker and P. A. Jacobs, Nature, 1990, 345-347.

13 S. Du, X. Chen, Q. Sun, N. Wang, M. Jia, V. Valtchev and J. Yu, Chem. Commun., 2016, 52, 3580-3583.

14 S. Du, F. Li, Q. Sun, N. Wang, M. Jia and J. Yu, Chem. Commun., 2016, 52, 3368-3371.

15 J. Zhou, Z. Hua, X. Cui, Z. Ye, F. Cui and J. Shi, Chem. Commun., 2010, 46, 4994-4996.

16 Y. Zuo, W. Song, C. Dai, Y. He, M. Wang, X. Wang and X. Guo, Appl. Catal., A, 2013, 453, 272-279.

17 A. Wróblewska, E. Makuch and P. Miądlicki, Catal. Today, 2016, 268, 121-129.

18 J. L. Grieneisen, H. Kessler, E. Fachea and A. M. L. Govic, Microporous Mesoporous Mater., 2000, 37, 379-386.

19 M. Taramasso, G. Perego and B. Notari, US Pat., US4410501, 1983.

20 U. Müller and W. Steck, Stud. Surf. Sci. Catal., 1994, 84, 203210.

21 G. Li, X. Wang, H. Yan, Y. Chen and Q. Su, Appl. Catal., A, 2001, 218, 31-38.
22 X. Wang, X. Guo and G. Li, Catal. Today, 2002, 74, 65-75.

23 M. Wang, J. Zhou, G. Mao and X. Zheng, Ind. Eng. Chem. Res., 2012, 39, 12730-12738.

24 T. Iwasaki, M. Isaka, H. Nakamura, M. Yasuda and S. Watano, Microporous Mesoporous Mater., 2012, 150, 1-6.

25 Q. Zhao, X. Hau, X. Liu, R. Zhai, L. Liu, X. Bao, X. Guo, G. Li and X. Wang, Mater. Chem. Phys., 2000, 66, 41-50.

26 P. Chen, X. Chen, X. Chen and H. Kita, J. Mater. Sci., 2009, 330, 369-378.

27 Q. H. Xia and Z. Gao, Mater. Chem. Phys., 1997, 47, 225-230.

28 X. Deng, Y. Wang, L. Shen, H. Wu, Y. Liu and M. He, Ind. Eng. Chem. Res., 2013, 52, 1190-1196.

29 D.-G. Huang, X. Zhang, T.-W. Liu, C. Huang, B.-H. Chen, C.-W. Luo, E. Ruckenstein and Z.-S. Chao, Ind. Eng. Chem. Res., 2013, 52, 3762-3772.

30 Y. Ding, Q. Ke, T. Liu, W. Wang, M. He, K. Yang, H. Jin, S. Wang and T. Tang, Ind. Eng. Chem. Res., 2014, 53, 13903-13909.

31 J. H. Zhang, M. B. Yue, X. N. Wang and D. Qin, Microporous Mesoporous Mater., 2015, 217, 96-101.

32 J. Wang, Y. Zhao, T. Yokoi, J. N. Kondo and T. Tatsum, ChemCatChem, 2014, 6, 2719-2726.

33 R. Millini, E. Previde Massara, G. Perego and G. Bellussi, J. Catal., 1992, 137, 497-503.

34 K. Fu, J. Yao, Q. Xiao, H. Liu, T. Li, T. Tatsumi and J.-G. Wang, RSC Adv., 2016, 6, 98514-98519.

35 W. Fan, R.-G. Duan, T. Yokoi, P. Wu, Y. Kubota and T. Tatsumi, J. Am. Chem. Soc., 2008, 10150-10164.

36 Q. Guo, K. Sun, Z. Feng, G. Li, M. Guo, F. Fan and C. Li, Chem.-Eur. J., 2012, 18, 13854-13860.

37 S. Bordiga, A. Damin, G. Berlier, F. Bonino, G. Ricchiardi, A. Zecchina and C. Lamberti, Int. J. Mol. Sci., 2001, 2, 167182.

38 J.-G. Wang, Y. Wang, T. Tatsumi and Y. Zhao, J. Catal., 2106, 338, 321-328.

39 M. S. Wendland and S. C. Zimmerman, J. Am. Chem. Soc., 1999, 121, 1389-1390.

40 S.-T. Tsai, P.-Y. Chao, T.-C. Tsai, I. Wang, X. Liu and X.-W. Guo, Catal. Today, 2009, 148, 174-178.

41 X. Wu, Y. Wang, T. Zhang, S. Wang, P. Yao, W. Feng, Y. Lin and J. Xu, Catal. Commun., 2014, 50, 59-62.

42 C. Hammond and G. Tarantino, Catalysts, 2015, 5, 23092323. 